\title{
Higher derivative terms in three dimensional supersymmetric theories
}

\author{
Adel Awad ${ }^{a, c}$ and Mir Faizal ${ }^{b}$ \\ ${ }^{a}$ Center for Theoretical Physics, British University of Egypt, \\ Sherouk City 11837, P.O. Box 43, Egypt \\ ${ }^{b}$ Department of Physics and Astronomy, University of Waterloo, \\ Waterloo, Ontario N2L 3G1, Canada \\ ${ }^{c}$ Department of Physics, Faculty of Science, \\ Ain Shams University, Abbassia, Cairo 11566, Egypt \\ E-mail: awad.adel@gmail.com, f2mir@uwaterloo.ca
}

ABSTRACT: In this work, we systematically analyze higher derivative terms in the supersymmetric effective actions for three dimensional scalar field theories using $\mathcal{N}=1$ superspace formalism. In these effective actions, we show that auxiliary fields do not propagate and their effective actions can be expressed in terms of the physical fields. So, the theory does not change its field content upon addition of higher derivative terms. We use derivative expansion to generate four, five and six dimensional terms for an interacting scalar field theory with $\mathcal{N}=1$ supersymmetry. We show that along with pure fermionic and bosonic terms, there are various five and six dimensional topological terms that mix bosonic and fermionic fields. Finally, we use these results to obtain higher derivative topological terms in the effective action for two M2-branes.

KEYWORDS: Superspaces, Supersymmetric Effective Theories

ARXIV EPRINT: 1503.08820 


\section{Contents}

1 Introduction 1

2 Auxiliary fields 3

3 Application 4

$\begin{array}{lll}4 & \text { Interacting theory } & 6\end{array}$

5 Action for M2-branes $\quad 8$

6 Topological terms for M2-branes $\quad 9$

7 Conclusion $\quad 12$

\section{Introduction}

Three dimensional supersymmetric field theories are interesting as they have been analyzed as examples of the $A d S_{4} / C F T_{3}$ correspondence in M-theory. The $\operatorname{OSp}(8 \mid 4)$ symmetry of the eleven dimensional supergravity on $A d S_{4} \times S_{7}$ is realized as $\mathcal{N}=8$ supersymmetry of the boundary superconformal field theory. This boundary superconformal field theory describes a system of multiple M2-branes. Furthermore, this boundary theory is constrained not to have any on-shell degrees of freedom coming from the gauge fields. All these properties are satisfied by BLG theory [28, 30-32, 34]. The BLG theory only describes two M2-branes. However, it has been possible to construct a generalization of the BLG theory called the ABJM theory [32-35]. The ABJM theory is thought to describe multiple M2-branes, and it reduces to the BLG theory for two M2-branes. Even though the ABJM theory has only $\mathcal{N}=6$ supersymmetry, it is expected that its supersymmetry might get enhanced to full $\mathcal{N}=8$ supersymmetry [36]. Just as the higher derivative correction to the D2-brane action can be written in form of Dirac-Born-Infeld action, it is possible to write higher derivative corrections to the ABJM theory. This can be done by writing the matter part of the ABJM theory in form of a gauge covariantized Nambu-Goto action. It may be noted that higher derivative corrections to this non-linear extension of the ABJM model have also been studied [37]. It has been shown that the Mukhi-Papageorgakis higgs mechanism can be used to determine higher derivative corrections to the BLG effective action [23]. This formalism is an on-shell formalism.

It may be noted that apart from the application to the physics of M2-branes and D2-branes, the addition of higher derivative corrections is interesting in its own right. Recently a generic three dimensional supersymmetric gauge theory coupled to matter fields has been constructed [38]. Under various limits this generic action reduces to the supersymmetric Maxwell theory, supersymmetric Maxwell-Chern-Simons, and supersymmetric 
Chern-Simons theories with matter fields. A generic three dimensional higher derivative superfield theory for self interacting scalar superfields has also been constructed [39]. In this analysis the self interacting higher derivative actions for real and complex scalar superfields have been studied.

Furthermore, supersymmetric theories with higher derivative terms play an important role in various cosmological models [1]. In the Dirac-Born-Infeld inflation, a scalar field describes the position of a brane plays the role of the inflaton field and causes an accelerated expansion of the universe $[2,3]$. The higher derivatives in the action cause new dynamics to arise and lead to equilateral-type non-gaussianity in the primordial density fluctuations [4]. Furthermore, higher derivatives play an important role even in ekpyrotic universes $[5,6]$. In these universes big bang is produced by the collision of branes moving in the bulk. In this model, a phase transition from a contracting phase to an expanding phase occurs for the ekpyrotic universes. This phase transition requires the violation of null energy condition. This conditions can be violated if the sum of the pressure and the effective energy density is negative. However, this leads to the existence of ghosts. It is possible to overcome this problem by adding higher derivative terms $[7,8]$. This occurs because of ghost condensation $[9,10]$.

It is known that certain higher derivative (HD) terms in effective actions of gauge theories with extended supersymmetries are not renormalized. The Seiberg-Dine terms with $\mathcal{N}=2$ supersymmetry [11, 12], and Wess-Zumino terms in the four dimensional superYang-Mills theory with $\mathcal{N}=4$ supersymmetry [13, 14], are examples of such terms. Furthermore, the HD terms generated from the D3-brane action have been used for analysing non-renormalization properties and anomalous dimensions of the four dimensional superYang-Mills theory with $\mathcal{N}=4$ supersymmetry. We hope to obtain similar results for the M2-branes, and this is one of the main motivations for this paper. It may be noted that the HD corrections for four dimensional field theories with $\mathcal{N}=1$ supersymmetry have already been studied [15-18]. Such terms have also been studied using the four dimensional harmonic superspace [24-27]. As M2-branes are described by a three dimensional superconformal field theory, we will analyse HD corrections to three dimensional supersymmetric field theories. So, in this work, we analyze HD terms generated in derivative expansion of three dimensional supersymmetric field theories in $\mathcal{N}=1$ superspace formalism.

In this work, we first consider the derivative expansion of an interacting supersymmetric scalar field theory. This analysis will be performed using $\mathcal{N}=1$ superspace formalism. It has been argued that the addition of HD terms in superspace formalism can cause problems in the original theory [19-22]. This is because a generic higher derivative action would contain terms like $\int_{\text {superspace }}(\partial \Phi)^{2} \sim \int_{\text {spacetime }}(\partial F)^{2}$, (where $F$ is the auxiliary field). This will produce kinetic terms for such auxiliary field. Thus, the HD terms will add new unwanted degrees of freedom to the original theory. Furthermore, in certain cases, these kinetic terms for the auxiliary field have the wrong sign, and this breaks the unitarity of the original theory. The vacuum of the theory can become unstable because of the HD terms. However, in this paper, we are able to explicitly demonstrate that the HD terms in the derivative expansion of a supersymmetric field theory, will only contain non-propagating auxiliary field. So, the action for this effective field theory can be always written in terms of physical fields. Therefore, the field content of the theory does not change upon adding 
HD terms. This is an important result as there has been a confusion regarding this point in earlier works [19-22].

Thus, using the superspace formalism, we obtain various four, five and six dimensional HD terms for an interacting scalar field theory. We show that pure fermionic and mixed topological terms with five and six mass dimensions exist in the effective action of this theory. Finally, we apply these results to obtain HD contributions to the effective field theory action for two M2-branes. We obtain several HD terms for two M2-branes, and we also compare them with an earlier study that has been done using the component fields.

The remaining paper is organized as follows. In section 2 , we use derivative expansion of the effective action of a real superfield in three dimensions to show that supersymmetric HD terms will not produce a kinetic energy term for the auxiliary field. In section 3, we apply this formalism to calculate the HD terms for a non-interacting supersymmetric scalar field theory. In section 4, we use derivative expansion to generate HD terms with mass dimensions four, five and six for an interacting supersymmetric theory. In section 5, we are going to review the construction of the BLG theory in $\mathcal{N}=1$ superspace formalism. In section 6, we are going to analyse the effective field theory action for two M2-branes. We will generate all six dimensional topological HD terms for two M2-branes. In the last section, we will summarize our results and discuss few extensions of this work.

\section{Auxiliary fields}

In this section, we use derivative expansion of the effective action of a real superfield in three dimensions to show that supersymmetric HD terms will not produce a kinetic energy term for the auxiliary field. So, the theory, even after the HD terms have been added to it, can still be expressed in terms of physical fields. We will apply these results to a non-interacting real scalar superfield theory.

Now before we present our argument for the supersymmetric theories, let us analyze the leading order HD terms for a non-supersymmetric theory. These terms will correct the kinetic energy term in the low energy effective action of the theory. A natural framework to study a set of HD terms in a particular theory is the derivative expansion of the low energy effective action, which reproduces the theory in the infrared limit. We will now perform such an expansion for a free massless scalar field $(\phi)$ theory in three dimensions. It is important to list the mass dimension of various fields, since in effective field theories, we consider the action up to a particular dimension, which are suppressed by some microscopic length scale $l$. Now from the kinetic terms of various fields, one can obtain the mass dimension for each field and derivative, $[\phi]=1 / 2,[\partial]=1,[m]=1$. It is important to state here that even if we are only interested in studying six dimensional terms, we have to include four and five dimensional terms for the consistency of the low energy effective action expansion.

Now consider a derivative expansion of a low-energy effective action of a real superfield $\Phi$ in a generic $\mathcal{N}=1$ supersymmetric theory ${ }^{1}$

$$
S_{t}=\int d^{2} \theta d^{3} x \mathcal{L}(\Phi, D \Phi, \ldots)
$$

\footnotetext{
${ }^{1}$ Here we use the notation of ref. [40].
} 
Since the length scale $l$ controls the derivative expansion, the leading order term has three mass dimensions. After integrating over the fermionic coordinates we get

$$
S_{t}=S_{0}+l S_{1}+l^{2} S_{2}+O\left(l^{3}\right)
$$

where $S_{n}=S_{n}(\phi, \psi, F) ; n=0,1,2, \ldots$ are functions of the component fields and have $n+3$ mass dimensions. Therefore, $S_{0}$ must be at most quadratic in the auxiliary field $F$, since $\Phi=\phi+\theta \psi-\theta^{2} F$, i.e., $[F]=3 / 2$. This means the general form of $S_{0}$ is given by

$$
S_{0}=\int d^{3} x\left[\frac{\alpha}{2} F^{2}+g(\phi, \psi) F+k(\phi, \psi)\right] .
$$

Now let us obtain the field equation of $F$, it reads

$$
S_{t}^{\prime}=S_{0}^{\prime}+l S_{1}^{\prime}+l^{2} S_{2}^{\prime}+O\left(l^{3}\right)=0
$$

where $S^{\prime}=\frac{\delta S}{\delta F}$. Since the microscopic scale $l$ controls the derivation expansion of the effective action and suppresses HD terms, it is natural to expand the fields in terms of $l$. Also all field fluctuations larger than $1 / l$ has been integrated out, therefore, the only field fluctuations, we have to consider should be less than $1 / l$. It is natural to expand $F$ in terms of $l$

$$
F=F_{0}+l F_{1}+l^{2} F_{2}+O\left(l^{3}\right) .
$$

Using equation (2.5) and expand $S_{1}^{\prime}$ and $S_{2}^{\prime}$ in terms of $l$, we get the following equations

$$
\begin{aligned}
\alpha F_{0}+g(\phi, \psi) & =0, \\
\alpha F_{1}+S_{1}^{\prime}\left(\phi, \psi, F_{0}\right) & =0, \\
\alpha F_{2}+S_{2}^{\prime}\left(\phi, \psi, F_{0}\right)+S_{1}^{\prime \prime}\left(\phi, \psi, F_{0}\right) F_{1} & =0 .
\end{aligned}
$$

These equations show that all the fields $F_{n}$ 's, can be all expressed as functions of the physical fields $\phi$ and $\psi$. Therefore, $F$ has no kinetic term, and the action can be written entirely in terms of $\phi$ and $\psi$. This result holds independent of the form of the HD terms in $S_{n}$. In fact, one can extend this argument to the four dimensional chiral and vector supersymmetric field theories in $\mathcal{N}=1$ superspace [29].

\section{Application}

Now we will apply the above argument to the supersymmetric action of a free massless real superfield $\Phi$ with higher derivative terms. Consider a real superfield $\Phi(x, \theta)=\phi+\theta^{a} \psi_{\alpha}-$ $\theta^{2} F[40]$, whose action is given by

$$
S_{0}=-\int d^{3} x d^{2} \theta\left[\frac{1}{2}\left(D^{\alpha} \Phi\right)^{2}\right] .
$$

In component form, the above action can be written as

$$
S_{0}=\int d^{3} x \frac{1}{2}\left[F^{2}+i \psi^{\alpha} \partial_{\alpha}{ }^{\beta} \psi_{\beta}+\phi \square \phi\right] .
$$


Using field equation of the auxiliary field $F=0$, which follows from this action, we can write the action of the component fields as,

$$
S_{0}=\int d^{3} x \frac{1}{2}\left[i \psi^{\alpha} \partial_{\alpha}{ }^{\beta} \psi_{\beta}+\phi \square \phi\right] .
$$

Now considering the above action with four and five dimensions HD terms, we obtain the following total action,

$$
S_{t}=\int d^{3} x d^{2} \theta\left[\Phi D^{2} \Phi+\alpha l\left(D^{2} \Phi\right)^{2}+\beta l^{2} D^{2} \Phi \square \Phi\right]+O\left(l^{3}\right),
$$

where $\alpha$ and $\beta$ are some couplings. Expanding the above action in component one obtains

$$
\begin{aligned}
S_{t}=\int d^{3} x \frac{1}{2}\left[F^{2}+\phi \square \phi+\right. & \left.i \psi^{\alpha} \partial_{\alpha}{ }^{\beta} \psi_{\beta}\right]+\alpha l\left[\psi^{\beta} \square \psi_{\beta}+2 F \square \phi\right] \\
& +\beta l^{2}\left[\phi \square^{2} \phi-i \psi_{\alpha} \partial^{\alpha}{ }_{\beta} \square \psi^{\beta}+F \square F\right]+O\left(l^{3}\right) .
\end{aligned}
$$

It may be noted that the auxiliary field has a kinetic term. Such terms occur in most supersymmetric field theories, if HD terms are considered. The field equation of the auxiliary field is given by

$$
F+2 \alpha l \square \phi+\beta l^{2} \square F=0 .
$$

It is natural to expand $F$ in terms of $l$

$$
F=F_{0}+l F_{1}+l^{2} F_{2}+O\left(l^{3}\right) .
$$

Using field equation, one gets,

$$
\begin{aligned}
& F_{0}=0, \\
& F_{1}=-2 \alpha \square \phi, \\
& F_{2}=-\beta \square F_{0}=0 .
\end{aligned}
$$

Now the total action reads

$$
\begin{aligned}
S_{t}=\int d^{3} x \frac{1}{2}\left[\phi \square \phi+i \psi^{\alpha} \partial_{\alpha}{ }^{\beta} \psi_{\beta}\right]+\alpha l \psi^{\beta} \square \psi_{\beta} \\
+l^{2}\left[\left(\beta-2 \alpha^{2}\right) \phi \square^{2} \phi-i \beta \psi_{\alpha} \partial^{\alpha}{ }_{\beta} \square \psi^{\beta}\right]+O\left(l^{3}\right) .
\end{aligned}
$$

It is not surprising that supersymmetry requires the existence of additional four dimension terms, and such terms did not exist in the non-supersymmetric version of the action. This is because the field content of the supersymmetric theory contains the fermionic field $\psi$. However, the interesting result here is that four dimension terms include an interesting fermionic topological term, $i \epsilon^{\mu \nu \sigma} \partial_{\mu} \psi_{\beta} \partial_{\nu} \psi^{\gamma}\left(\gamma_{\sigma}\right)_{\gamma}^{\beta}$. Even though, it is a total derivative for four mass-dimension terms, it can be argued that it is possible to have a five dimensional topological term in an interacting theory. Furthermore, in the coming section, we show the possibility of having a pure fermionic topological term with six mass dimensions of the form, $\psi^{2} \epsilon^{\mu \nu \sigma} \partial_{\mu} \psi_{\beta} \partial_{\nu} \psi^{\gamma}\left(\gamma_{\sigma}\right)_{\gamma}^{\beta}$. 


\section{Interacting theory}

Now we will analyze higher derivative terms for the interacting supersymmetric $\phi^{6}$ theory, which is renormalizable in three dimensions. The supersymmetric action for this theory can be written as

$$
S_{0}=\int d^{3} x d^{2} \theta\left[-\frac{1}{2}\left(D^{\alpha} \Phi\right)^{2}+\frac{\lambda}{4 !} \Phi^{4}\right] .
$$

In component form, the above action can be written as

$$
S_{0}=\int d^{3} x \frac{1}{2}\left[F^{2}+i \psi^{\alpha} \partial_{\alpha}{ }^{\beta} \psi_{\beta}+\phi \square \phi\right]+\frac{\lambda}{2} \phi^{2} \psi^{2}+\frac{\lambda}{3 !} F \phi^{3} .
$$

Using the field equation for the auxiliary field, $F=-\lambda \phi^{3} / 3$ !, we obtain

$$
S_{0}=\int d^{3} x \frac{1}{2}\left[i \psi^{\alpha} \partial_{\alpha}{ }^{\beta} \psi_{\beta}+\phi \square \phi\right]+\frac{\lambda}{2} \phi^{2} \psi^{2}-\frac{\lambda^{2}}{2(3 !)^{2}} \phi^{6} .
$$

Now we will write the low energy effective theory with all possible HD terms consistent with the symmetries up to dimension five. The list of independent four and five dimensional terms is given by

$$
\begin{gathered}
S_{1}=l \int d^{3} x d^{2} \theta\left[c_{1}\left(D^{2} \Phi\right)^{2}+c_{2} \Phi^{2}\left(D_{\alpha} \Phi\right)^{2}+c_{4} \Phi^{6}\right], \\
S_{2}=l^{2} \int d^{3} x d^{2} \theta\left[c_{5} D^{2} \Phi \square \Phi+c_{6} \Phi^{2}\left(D^{2} \Phi\right)^{2}+c_{7} \Phi D^{2} \Phi\left(D_{\alpha} \Phi\right)^{2}\right. \\
\left.+c_{8}\left(D_{\alpha} \Phi\right)^{4}+c_{9} \Phi^{4}\left(D_{\alpha} \Phi\right)^{2}+c_{10} \Phi^{8}\right] .
\end{gathered}
$$

Now we can write all the four dimensional terms in component fields as

$$
\begin{aligned}
\int d^{2} \theta\left(D^{2} \Phi\right)^{2} & =\psi_{\alpha} \square \psi^{\alpha}+2 F \square \phi, \\
\int d^{2} \theta \Phi^{2}\left(D_{\alpha} \Phi\right)^{2} & =\phi^{2}\left[F^{2}+i \psi^{\alpha} \partial_{\alpha}{ }^{\beta} \psi_{\beta}+\phi \square \phi\right]+\psi^{4}+2 \psi^{2} \phi F, \\
\int d^{2} \theta \Phi^{6} & =30 \psi^{2} \phi^{4}+6 F \phi^{5} .
\end{aligned}
$$

We can also write all the five dimensional terms in component fields as

$$
\begin{aligned}
\int d^{2} \theta\left(D^{2} \Phi\right) \square \Phi= & \phi \square^{2} \phi-i \psi_{\alpha} \partial_{\beta}^{\alpha} \square \psi^{\beta}+F \square F, \\
\int d^{2} \theta\left(D^{2} \Phi\right)^{2} \Phi^{2}= & \phi^{2}\left[\psi^{\beta} \square \psi_{\beta}+i \epsilon^{\mu \nu \sigma} \partial_{\mu} \psi_{\beta} \partial_{\nu} \psi^{\gamma}\left(\gamma_{\sigma}\right)_{\gamma}^{\beta}+2 F \square \phi\right]+2 \phi F^{3} \\
& +2 \psi^{2} F^{2}+i 8 \phi \psi_{\alpha} \partial_{\beta}^{\alpha} \psi^{\beta} F, \\
\int d^{2} \theta\left(D^{2} \Phi\right) \Phi\left(D_{\alpha} \Phi\right)^{2}= & F^{2} \psi^{2}-i \psi^{2} \psi^{\alpha} \partial_{\alpha}^{\beta} \psi_{\beta}+\phi \square \phi \psi^{2}+i \phi F \psi^{\alpha} \partial_{\alpha}^{\beta} \psi_{\beta}-\phi \psi^{\gamma} \partial_{\mu} \psi_{\gamma} \partial^{\mu} \phi \\
& +i \epsilon^{\mu \nu \sigma} \phi \psi^{\beta}\left(\gamma_{\sigma}\right)_{\beta}^{\alpha} \partial_{\mu} \psi_{\alpha} \partial_{\nu} \phi, \\
\int d^{2} \theta\left(D_{\alpha} \Phi\right)^{4}= & \psi^{2}\left[\partial_{\mu} \phi \partial^{\mu} \phi+2 F^{2}\right], \\
\int d^{2} \theta \Phi^{4}\left(D_{\alpha} \Phi\right)^{2}= & 12 \psi^{4} \phi^{2}+4 \psi^{2} \phi^{3} F+8 \psi^{2} \phi^{3} F+\phi^{4}\left[F^{2}+i \psi^{\alpha} \partial_{\alpha}{ }^{\beta} \psi_{\beta}+\phi \square \phi\right], \\
\int d^{2} \theta \Phi^{8}= & 56 \psi^{2} \phi^{6}+8 \phi^{7} F .
\end{aligned}
$$


The term $\psi_{\beta}\left(\gamma_{\sigma}\right)_{\gamma}^{\beta} \psi^{\gamma} \epsilon^{\mu \nu \sigma} \partial_{\mu} \phi \partial_{\nu} \phi$ mixes the scalars and fermions in five dimensional terms. Usually such topological terms are are non-renormalizable. This makes them an important tool to study the non-perturbative nature of the theory. Although, this term vanishes identically for a single scalar superfield $\Phi$, we will show in the next section that with more than one superfield (i.e., $\Phi^{I}$ is in some representation of certain group $G$ ) these terms become non-vanishing.

Now the list of six dimension terms in supersymmetric $\phi^{6}$ theory is given by

$$
\begin{aligned}
S_{3}=l^{3} \int d^{3} x & d^{2} \theta\left[c_{11}\left(D^{2} \Phi\right)^{3} \Phi+c_{12}\left(D^{2} \Phi\right)^{2} \Phi^{4}+c_{13}\left(D^{2} \Phi\right) \Phi^{7}\right. \\
& +c_{14}\left(D_{\alpha} \Phi\right)^{2} D^{2} \Phi \Phi^{3}+c_{15}\left(D_{\alpha} \Phi\right)^{2}\left(D^{2} \Phi\right)^{2} \\
& \left.+c_{16} D^{2} \Phi(\square \Phi) \Phi^{2}+c_{17}(\square \Phi)^{2}+c_{18}(\square \Phi) \Phi^{5}+c_{19} \Phi^{10}\right] .
\end{aligned}
$$

We can write all the six dimensional terms in component fields as

$$
\begin{aligned}
\int d^{2} \theta\left(D^{2} \Phi\right)^{3} \Phi= & 3 F^{2}\left[i \psi^{\alpha} \partial_{\alpha}^{\beta} \psi_{\alpha}+F^{2}+\phi \square \phi\right]-6 \phi F \partial_{\mu} \psi^{\alpha} \partial^{\mu} \psi_{\alpha} \\
& +i 6 \phi F \epsilon^{\mu \nu \sigma} \partial_{\mu} \psi^{\alpha}\left(\gamma_{\sigma}\right)_{\alpha \beta} \partial_{\nu} \psi^{\beta}, \\
\int d^{2} \theta\left(D^{2} \Phi\right)^{2} \Phi^{4}= & 2 F^{3} \Phi^{3}+3 \psi^{2} \phi^{2} F^{2}+i 8 \psi_{\alpha} \partial_{\beta}^{\alpha} \psi^{\beta} \phi^{3} F+2 \phi^{4} F \square \phi \\
& -\frac{1}{2} \phi^{4} \partial_{\mu} \psi^{\beta} \partial^{\mu} \psi_{\beta}-\frac{i}{2} \phi^{4} \epsilon^{\mu \nu \sigma}\left(\gamma_{\sigma}\right)_{\alpha}^{\beta} \partial_{\mu} \psi_{\beta} \partial_{\nu} \psi^{\alpha}+4 i F \phi^{3} \psi^{\alpha} \partial_{\alpha}^{\beta} \psi_{\beta}, \\
\int d^{2} \theta\left(D^{2} \Phi\right) \Phi^{7}= & \phi^{7} \square \phi-7 i \phi^{6} \psi^{\alpha} \partial_{\alpha}^{\beta} \psi_{\beta}+7 F^{2} \phi^{6}+42 \psi^{2} F \phi^{5}, \\
& +\frac{1}{4} \phi^{3} \psi_{\beta} \partial_{\mu} \psi^{\beta} \partial^{\mu} \phi+\frac{i}{4} \phi^{3} F \psi^{\alpha} \partial_{\alpha}^{\beta} \psi_{\beta}+3 i \phi^{2} \psi^{2} \psi^{\alpha} \partial_{\alpha}^{\beta} \psi_{\beta} \\
& -2 F^{3} \phi^{3}+F \phi^{3} \partial_{\mu} \phi \partial^{\mu} \phi+\frac{3}{2} F^{2} \psi^{2} \phi^{2}+6 F \psi^{4} \phi, \\
d^{2} \theta\left(D_{\alpha} \Phi\right)^{2}\left(D^{2} \Phi\right) \Phi^{3}= & \phi^{3} \square \phi \psi^{2}-\frac{i}{4} \phi^{3} \epsilon^{\mu \nu \sigma}\left(\gamma_{\sigma}^{\beta} \partial_{\mu} \psi_{\beta} \partial_{\nu} \psi^{\alpha}\right. \\
\int d^{2} \theta\left(D_{\alpha} \Phi\right)^{2}\left(D^{2} \Phi\right)^{2}= & F \psi^{2} \square \phi-\frac{i}{2} \psi^{2}\left[\partial_{\mu} \psi_{\alpha} \partial^{\mu} \psi^{\alpha}+i \epsilon^{\mu \nu \sigma}\left(\gamma_{\sigma}\right)_{\alpha}^{\beta} \partial_{\mu} \psi_{\beta} \partial_{\nu} \psi^{\alpha}\right] \\
& -\frac{3}{2} F^{2} \psi^{\alpha} \partial_{\alpha}^{\beta} \psi_{\beta}+\frac{3}{2} F\left[\partial_{\mu} \psi_{\alpha} \partial^{\mu} \phi \psi^{\alpha}+i \epsilon^{\mu \nu \sigma}\left(\gamma_{\sigma}\right)_{\alpha}^{\beta} \partial_{\mu} \psi_{\beta} \partial_{\nu} \phi \psi^{\alpha}\right], \\
\int d^{2} \theta\left(D^{2} \Phi\right) \square \Phi \Phi^{2}= & (\square \phi)^{2} \phi^{2}+2 i \psi_{\alpha} \partial_{\beta}^{\alpha} \psi^{\beta} \phi \square \phi+F \square F \phi^{2}+2 \phi F \psi^{\beta} \square \psi_{\beta} \\
& +F \square F \phi^{2}+2 F^{2} \phi \square \phi-2 F \psi^{2} \square \phi, \\
\int d^{2} \theta(\square \Phi) \Phi^{5}= & 2 \phi^{5} \square F+5 \phi^{4} \psi_{\alpha} \square \psi^{\alpha}+5 F \phi^{4} \square \phi+20 \phi^{3} \psi^{2} \square \phi . \\
\int d^{2} \theta(\square \Phi)^{2}= & 2 \square F \square+\square \psi_{\alpha} \square \psi^{\alpha}, \\
d^{2} \theta(\Phi)^{10}= & \frac{45}{2} \phi^{8} \psi^{2}+10 F \phi^{9},
\end{aligned}
$$

Notice the existence of non-vanishing purely fermionic topological terms as well as mixed topological terms. With more than one superfield, these terms should play an important role in the effective action of M2-branes. 
As we have mentioned before, having a single superfield forces certain topological terms to vanish as a result of anti-symmetrization of the spacetime derivatives. Let us list few examples of those terms keeping in mind that some of them (dimension 6 terms) will appear in the following section. Thus, we can write the five dimensions term as

$$
\begin{aligned}
\int d^{2} \theta\left(D_{\beta} \Phi\right)\left(D^{\beta} D_{\rho} \Phi\right)\left(D^{\rho} \Phi\right) \Phi \\
\supset \epsilon^{\mu \nu \sigma} \partial_{\mu} \phi \partial_{\nu} \phi \psi^{\beta}\left(\gamma_{\sigma}\right)_{\beta}^{\alpha} \psi_{\alpha}+\epsilon^{\mu \nu \sigma} \phi \partial_{\mu} \phi \partial_{\nu} \phi \partial_{\sigma} \phi,
\end{aligned}
$$

and the six dimensional term as

$$
\begin{aligned}
& \int d^{2} \theta \Phi^{3}\left(D_{\beta} \Phi\right)\left(D^{\beta} D_{\rho} \Phi\right)\left(D^{\rho} \Phi\right) \\
& \supset \epsilon^{\mu \nu \sigma} \phi^{2} \partial_{\mu} \phi \partial_{\nu} \phi \psi^{\beta}\left(\gamma_{\sigma}\right)_{\beta}^{\alpha} \psi_{\alpha}+\epsilon^{\mu \nu \sigma} \phi^{3} \partial_{\mu} \phi \partial_{\nu} \phi \partial_{\sigma} \phi .
\end{aligned}
$$

\section{Action for M2-branes}

In this section, we are going to review the construction of the action for two M2-branes. The BLG theory describes the physics of two M2-branes. So, here we review the construction of the BLG theory in $\mathcal{N}=1$ superspace formalism [41]. The gauge fields in the BLG theory are valued in a Lie 3-algebra rather than a conventional Lie algebra. A Lie 3-algebra is a vector space endowed with a trilinear product,

$$
\left[T^{a}, T^{b}, T^{c}\right]=f_{d}^{a b c} T^{d}
$$

The structure constants of this Lie 3 -algebra are totally antisymmetric in $a, b, c$. They also satisfy the Jacobi identity [42],

$$
f_{g}^{[a b c} f_{h}^{d] e g}=0 .
$$

The metric of this Lie 3-algebra can be defined by taking the trace over the Lie 3-algebra indices,

$$
h^{a b}=\operatorname{Tr}\left(T^{a} T^{b}\right) .
$$

It is also possible to define a symmetrised trace of four Lie 3-algebra generators as

$$
\operatorname{Str}\left(T^{a} T^{b} T^{c} T^{d}\right)=m h^{(a b} h^{c d)},
$$

where $m$ is a constant. For the Lorentz Lie 3 -algebra, it is possible to consider a set of generators corresponding to a compact subgroup of the full symmetry group. Hence, we can choose the generators of a $\mathrm{SU}(2)$ Lie algebra, and write [23]

$$
\begin{aligned}
\operatorname{Tr}\left(T^{a} T^{b}\right) & =\frac{1}{2} \delta^{a b}, \\
S T r\left(T^{a} T^{b} T^{c} T^{d}\right) & =\frac{1}{4} \delta^{(a b} \delta^{c d)} .
\end{aligned}
$$

The gauge fields are valued in the Lie 3-algebra, $\Gamma_{a b}^{\alpha} T^{a} T^{b}=\Gamma^{\alpha}$. The BLG theory has been written using $\mathcal{N}=1$ superspace formalism. This is done by writing defining 
$\Phi^{I}{ }_{a}=\phi^{I}{ }_{a}+\theta^{\alpha} \psi_{\alpha a}^{I}-\theta^{2} F^{I}{ }_{a}$, with $I=1,2, \ldots 8$, where $a$ is the three-algebra index with a structure constant $f^{a b c d}$. So, we can write the action for the BLG theory as [41]

$$
\begin{aligned}
S_{0}=-\int d^{3} x d^{2} \theta & {\left[\frac{1}{4}\left(D^{\alpha} \Phi_{d}^{I}+f_{d}^{a b c} \Gamma_{a b}^{\alpha} \Phi_{c}^{I}\right)^{2}+\frac{1}{8} f^{a b c d}\left(D^{\alpha} \Gamma_{a b}^{\beta}\right)\left(D_{\beta} \Gamma_{\alpha c d}\right)\right.} \\
+ & \left.\frac{1}{6} f_{g}^{c d a} f^{e f g b}\left(D^{\alpha} \Gamma_{a b}^{\beta}\right) \Gamma_{\alpha c d} \Gamma_{\beta e f}+\frac{1}{24} f^{a b c d} C_{I J K L} \Phi_{a}^{I} \Phi_{b}^{J} \Phi_{c}^{K} \Phi_{d}^{L}\right] .
\end{aligned}
$$

The component field definitions are as

$$
\begin{array}{rlrl}
\Phi_{a}^{I} \mid & =\phi_{a}^{I}, & D_{\alpha} \Phi_{a}^{I} \mid & =\psi_{\alpha a}^{I}, \\
\Gamma_{\alpha a b} \mid & =\chi_{\alpha a b}, \\
\frac{1}{2} \Phi_{a}^{I} \mid- & =F_{a}^{I}, & \Gamma_{\alpha a b} \mid & =2 \lambda_{\alpha a b}-i \partial_{\alpha}^{\beta} \chi_{\beta a b}, \\
D_{\alpha a b} \mid & =B_{a b}, & D^{2} \Gamma_{a b}^{\alpha} \mid & =2 \lambda_{a b}^{\alpha}+i \partial_{\beta}^{\alpha} \chi_{a b}^{\beta}, \\
D_{\alpha} \Gamma_{a b}^{\beta} \mid & =i\left(\gamma_{\mu}\right)_{\alpha}^{\beta} A_{a b}^{\mu}-\delta_{\alpha}^{\beta} B_{a b}, & \frac{1}{2} D^{\beta} D_{\alpha} \Gamma_{\beta a b} \mid & =\lambda_{\alpha a b} . \\
D^{\alpha} \Gamma_{\beta a b} \mid & =i\left(\gamma_{\mu}\right)_{\beta}^{\alpha} A_{a b}^{\mu}+\delta_{\beta}^{\alpha} B_{a b}, &
\end{array}
$$

An octonion algebra $\left\{1, e_{i}\right\}$, with $i=1, \ldots, 7$, such that $e_{i} e_{j}=c_{i j k} e_{k}-\delta_{i j}$, has been used to defined $C_{I J K L}$. This is done by taking a totally antisymmetric tensor $c_{i j k}$. The seven dimensional dual of this is $c_{i j k l}=\frac{1}{6} \epsilon_{i j k l m n o} c^{m n o}$. Now it is possible to construct an $\mathrm{SO}(7)$ invariant tensor $C_{I J K L} I, J, K, L=1, \ldots, 8$ which is self dual in eight dimensions, $C_{i j k 8}=$ $c_{i j k}, C_{i j k l}=c_{i j k l}$. This octonionic structure constants can be used to construct $\mathrm{SO}(8)$ gamma matrices [41]. So, we can use write $\left(\Gamma^{i}\right)_{A \dot{A}}=c_{A \dot{A}}^{i}+\delta_{8 \dot{A}} \delta_{A i}-\delta_{8 A} \delta_{\dot{A} i}$, where $i=1, \ldots, 7$ and $A, \dot{A}=1, \ldots, 8$. We also have $\left(\Gamma^{8}\right)_{A \dot{A}}=\delta_{A \dot{A}} c_{8 \dot{A}}^{i}=c_{A 8}^{i}=0$. Here we have defined $\hat{\Gamma}_{\dot{A} A}^{I}=\left(\Gamma^{T}\right)_{\dot{A} A}^{I}$, and $\Gamma^{I} \hat{\Gamma}^{J}+\Gamma^{J} \hat{\Gamma}^{I}=2 \delta^{I J}$. Now the clifford algebra can be written as

$$
\gamma^{I} \gamma^{J}+\gamma^{J} \gamma^{I}=2 \delta^{I J}
$$

where

$$
\gamma^{I}=\left(\begin{array}{cc}
0 & \Gamma_{A \dot{A}}^{I} \\
\hat{\Gamma}_{\dot{A} A}^{I} & 0
\end{array}\right)
$$

Now we can also write

$$
\Gamma_{A B}^{I J}=\frac{1}{2}\left(\Gamma_{A \dot{A}}^{I} \hat{\Gamma}_{\dot{A} B}^{J}-\Gamma_{A \dot{A}}^{J} \hat{\Gamma}_{\dot{A} B}^{I}\right)=C_{A B}^{I J}+\delta_{A}^{I} \delta_{B}^{J}-\delta_{B}^{I} \delta_{A}^{J} .
$$

\section{Topological terms for M2-branes}

In this section, we will analyse the effective action for two M2-branes. We will use the above analysis to argue for the existence of new topological HD terms in the effective action of M2-branes. The effective M2-brane action can be expanded in terms of Planck length $l_{p}$ as follows

$$
S_{B L G}=S_{0}+l_{p}^{3} S_{3}+\ldots
$$


Therefore, the first correction to the leading contribution is of dimension six. This is why we have expanded our effective action in the general $\phi^{6}$ theory up to such order. The action of the theory without the gauge field, i.e., the Higgs branch, is given by

$$
S_{0}=-\int d x^{3} d^{2} \theta \operatorname{Tr}\left(\left(D_{\alpha} \Phi\right)^{2}+\frac{1}{12}\left[\Phi^{I}, \Phi^{J}, \Phi^{K}\right] \Phi^{L} C_{I J K L}\right)
$$

Since the leading correction is of order $O\left(l_{p}^{3}\right)$, the field equation for the auxiliary field is given by

$$
F_{a}^{I}=\frac{-1}{6} f^{a b c d} C_{J K L}^{I} \Phi_{b}^{J} \Phi_{c}^{K} \Phi_{d}^{L}+O\left(l_{p}^{3}\right)
$$

The non-vanishing six dimensional topological terms can be classified as bosonic, fermionic and mixed terms. Here we list all such terms. We find the following bosonic terms,

$$
\begin{aligned}
\mathcal{L}_{b 1}= & \int d^{2} \theta S \operatorname{ST}\left(C_{I J K L} C_{J^{\prime} K^{\prime} L^{\prime}}^{I} \Phi^{J^{\prime} K^{\prime} L^{\prime}} D_{\beta} \Phi^{J} D^{\beta} D_{\gamma} \Phi^{K} D^{\gamma} \Phi^{L}\right) \\
\supset S T r & \left(C_{I J K L} C_{J^{\prime} K^{\prime} L^{\prime}}^{I} \phi^{J^{\prime} K^{\prime} L^{\prime}} \epsilon^{\mu \nu \sigma} \partial_{\mu} \phi^{J} \partial_{\sigma} \phi^{K} \partial_{\nu} \phi^{L}\right) \\
= & \frac{1}{4} C_{I J K L} C_{J^{\prime} K^{\prime} L^{\prime}}^{I} \phi_{a^{\prime} b^{\prime} c^{\prime}}^{J^{\prime} L^{\prime}} f^{a^{\prime} b^{\prime} c^{\prime}}{ }_{d} \epsilon^{\mu \nu \sigma} \times \partial_{\mu} \phi_{a}^{J} \partial_{\sigma} \phi_{b}^{K} \partial_{\nu} \phi_{c}^{L} \delta^{(a b} \delta^{c d)}
\end{aligned}
$$

Another term that produces the same topological term is given by

$$
\begin{aligned}
\mathcal{L}_{b 1^{\prime}}=\int d^{2} \theta \operatorname{STr}\left(C_{I J K L} D^{2} \Phi^{I} D_{\beta} \Phi^{J} D^{\beta} D_{\gamma} \Phi^{K} D^{\gamma} \Phi^{L}\right) \\
\supset S \operatorname{ST}\left(C_{I J K L} F^{I} \epsilon^{\mu \nu \sigma} \partial_{\mu} \phi^{J} \partial_{\sigma} \phi^{K} \partial_{\nu} \phi^{L}\right) .
\end{aligned}
$$

The relation between this term and the previous one is clear upon using eq. (6.3). This term has also been obtained in earlier works on M2-branes [23]. We also find three different fermionic terms

$$
\begin{gathered}
\mathcal{L}_{f 1}=\int d^{2} \theta \operatorname{Tr}\left(C^{I J K L} D^{\alpha} \Phi^{I}\left[D_{\alpha} \Phi^{J}, D^{2} \Phi^{K}, D^{2} \Phi^{L}\right]\right) \\
\supset C^{I J K L} f^{a b c d} \epsilon^{\mu \nu \sigma} \psi_{a}^{I} \cdot \psi_{b}^{J} \partial_{\mu} \psi_{c}^{K} \cdot \gamma_{\nu} \cdot \partial_{\sigma} \psi_{d}^{L} \\
\mathcal{L}_{f 2}=\int d^{2} \theta \operatorname{STr}\left(D^{\alpha} \Phi^{I} D_{\alpha} \Phi^{J} D^{2} \Phi^{I} D^{2} \Phi^{J}\right) \\
\supset \delta^{(a b} \delta^{c d)} \epsilon^{\mu \nu \sigma} \psi_{a}^{I} \cdot \psi_{b}^{J} \partial_{\mu} \psi_{c}^{I} \cdot \gamma_{\nu} \cdot \partial_{\sigma} \psi_{d}^{J} \\
\mathcal{L}_{f 3}=\int d^{2} \theta \operatorname{STr}\left(D^{\alpha} \Phi^{I} D_{\alpha} \Phi^{I} D^{2} \Phi^{J} D^{2} \Phi^{J}\right) \\
\supset \delta^{(a b} \delta^{c d)} \epsilon^{\mu \nu \sigma} \psi_{a}^{I} \cdot \psi_{b}^{I} \partial_{\mu} \psi_{c}^{J} \cdot \gamma_{\nu} \cdot \partial_{\sigma} \psi_{d}^{J},
\end{gathered}
$$

where $\psi \cdot \xi=\frac{1}{2} \psi^{\alpha} \xi_{\alpha}$ and $\psi \cdot \gamma^{\mu} \cdot \xi=\psi_{\alpha}\left(\gamma^{\mu}\right)^{\alpha \beta} \xi_{\beta}$. This last term can be expressed in the 
notation of [23] as $\operatorname{Tr}\left(\bar{\Psi} \Psi \epsilon^{\mu \nu \sigma} D_{\mu} \bar{\Psi} \gamma_{\nu} D_{\sigma} \Psi\right)$. Finally, we find the following mixed terms,

$$
\begin{aligned}
& \mathcal{L}_{m 1}= \int d^{2} \theta \operatorname{Tr}\left(D_{\gamma} D_{\alpha} \Phi^{J}\left[\Phi^{I}, D_{\beta} \Phi^{J}, D^{\beta} D^{\gamma} D^{\alpha} \Phi^{I}\right]\right) \\
& \supset f^{a b c d} \epsilon^{\mu \nu \sigma} \phi_{a}^{I} \phi_{b}^{J} \partial_{\mu} \psi_{c}^{I} \cdot \gamma_{\nu} \cdot \partial_{\sigma} \psi_{d}^{J} \\
& \mathcal{L}_{m 2}= \int d^{2} \theta S \operatorname{Tr}\left(C_{I J K L} D_{\gamma} D_{\alpha} \Phi^{I} \Phi^{J} D_{\beta} \Phi^{K} D^{\beta} D^{\gamma} D^{\alpha} \Phi^{L}\right) \\
& \supset C_{I J K L} \delta^{(a b} \delta^{c d)} \epsilon^{\mu \nu \sigma} \phi_{a}^{I} \phi_{b}^{J} \partial_{\mu} \psi_{c}^{K} \cdot \gamma_{\nu} \cdot \partial_{\sigma} \psi_{d}^{L} \\
& \mathcal{L}_{m 3}= \int d^{2} \theta \operatorname{Tr}\left(C^{I J K}{ }_{L} C_{I^{\prime} J^{\prime} K^{\prime}}{ }^{L} \Phi^{I^{\prime} J^{\prime} K^{\prime}}\left[\Phi^{I}, D^{\alpha} \Phi^{J}, D^{2} D_{\alpha} \Phi^{K}\right]\right) \\
& \supset C^{I J K}{ }_{L} C_{I^{\prime} J^{\prime} K^{\prime}}{ }^{L} \phi_{a^{\prime} b^{\prime} c^{\prime}}^{I^{\prime} J^{\prime} K^{\prime}} f^{a^{\prime} b^{\prime} c^{\prime} d} f_{d}^{a b c} \epsilon^{\mu \nu \sigma} \\
& \\
& \times \phi_{a}^{I} \partial_{\mu} \psi_{c}^{J} \cdot \gamma_{\nu} \cdot \partial_{\sigma} \psi_{d}^{K}
\end{aligned}
$$

The same topological term can be produced by

$$
\begin{aligned}
\mathcal{L}_{m 3^{\prime}}=\int d^{2} \theta \operatorname{Tr}\left(C^{I J K}{ }_{L} D^{2} \Phi^{L}\left[\Phi^{I}, D^{\alpha} \Phi^{J}, D^{2} D_{\alpha} \Phi^{K}\right]\right) \\
\supset C^{I J K}{ }_{L} F_{d}^{L} f^{a b c}{ }_{d}^{\mu \nu \sigma} \phi_{a}^{I} \partial_{\mu} \psi_{c}^{J} \cdot \gamma_{\nu} \cdot \partial_{\sigma} \psi_{d}^{K}
\end{aligned}
$$

The last mixed term can be written as

$$
\begin{aligned}
\mathcal{L}_{m 4}= & \int d^{2} \theta S T r\left(C_{I^{\prime} J^{\prime} K^{\prime}}^{I} \Phi^{I^{\prime} J^{\prime} K^{\prime}} \Phi^{J} D^{\alpha} \Phi^{I} D^{2} D_{\alpha} \Phi^{J}\right) \\
& \supset C_{I^{\prime} J^{\prime} K^{\prime}}{ }^{I} \phi_{a^{\prime} b^{\prime} c^{\prime}}^{I^{\prime} K^{\prime} K^{\prime}} f^{a^{\prime} b^{\prime} c^{\prime}}{ }_{d} \delta^{(a b} \delta^{c d)} \epsilon^{\mu \nu \sigma} \\
& \times \phi_{a}^{J} \partial_{\mu} \psi_{b}^{I} \cdot \gamma_{\nu} \cdot \partial_{\sigma} \psi_{c}^{J}
\end{aligned}
$$

Another term that produces the same topological term, after using eq. (6.3), takes the form

$$
\begin{aligned}
\mathcal{L}_{m 4^{\prime}}=\int d^{2} \theta \operatorname{STr}\left(D^{2} \Phi^{I} \Phi^{J} D^{\alpha} \Phi^{I} D^{2} D_{\alpha} \Phi^{J}\right) \\
\\
\supset \delta^{(a b} \delta^{c d)} F_{d}^{I} \epsilon^{\mu \nu \sigma} \phi_{a}^{J} \partial_{\mu} \psi_{b}^{I} \cdot \gamma_{\nu} \cdot \partial_{\sigma} \psi_{c}^{J} .
\end{aligned}
$$

It is worth mentioning here that these six dimensional HD terms of the M2-branes effective action have been calculated in earlier studies [23]. This was done by using a novel Higgs mechanism, and this reduced the M2-brane action to a matter-Yang-Mills theory describing the low energy effective action of multiple D2-branes. The HD terms obtained in this work are written in terms of component fields, and the lowest order field equations in derivative expansion was used. This made several of these HD terms to vanish. In contrast to this, the HD terms in this work have been constructed using superspace formalism, and they have been written in terms of $\mathcal{N}=1$ superfields. This explains why all these terms were not obtained in earlier studies [23]. In fact, in the earlier component formalism only the pure bosonic topological term was obtained. Apart from this pure bosonic terms, all the HD terms produced here using the superspace formalism, vanished upon using lowest order field equations in derivative expansion. The reason is that these terms contain fermions and can be written in terms of one or more factors of $i \partial_{\alpha \beta} \psi^{\beta}$, which are set to zero by the lowest order field equation in derivative expansion. This explains why these terms were absent 
in earlier studies [23]. The importance of these HD terms come from the fact that they modify the interaction Lagrangian, therefore, they affect loop calculations of scattering amplitudes in the low energy effective theory $[43,44]$. In addition, if we consider path integral quantization for such an effective theory we have to sum over all such HD terms in our Lagrangian, otherwise we will not have the correct interaction Lagrangian $[45,46]$.

\section{Conclusion}

In this paper, we have analyzed the higher derivative terms for three dimensional supersymmetric theories with $\mathcal{N}=1$ supersymmetry. We first analyzed the higher derivative terms for a general scalar superfield theory and demonstrated that the auxiliary field will not acquire a kinetic term for all possible actions of the theory. Therefore, the theory is completely describable in terms of its original field content. We calculated all four, five and six dimensional terms for such a theory demonstrating the existence of various interesting topological terms. We obtained pure bosonic, pure fermionic and terms which mix bosonic and fermionic fields. We also analyse the effective action for the BLG theory in $\mathcal{N}=1$ superspace formalism. We show the existence of several mixed and pure fermionic terms which vanish upon using the lowest order field equations in derivative expansion. These terms were absent in the list of six dimensional terms generated in earlier studies [23]. It may be noted that even though these terms vanish upon using the lowest order field equations in derivative expansion, it is important to consider them, as they can affect loop calculations of scattering amplitudes in the low energy effective theory.

It will be interesting to generalize the results of this paper, for theories with higher amount of supersymmetry. Furthermore, it will be interesting to perform a similar analysis for matter fields coupled to gauge fields. The results thus obtained can be used for analyzing HD corrections to the M2-branes effective actions using the ABJM theory. It is possible to extend this work done on global supersymmetry with higher derivative terms to local supergravity theories. In fact, the supergravity extension of scalar field theories with higher derivative terms has been studied [47]. This analysis was done using supergravity in $\mathcal{N}=1$ superspace formalism. The elimination of auxiliary fields modifies both the kinetic and potential terms in this theory. In this case, it has been demonstrated that potential energy can be generated even if there was no original superpotential term in the action. It will be interesting to extend the results of this paper to local supergravity theories in three dimensions.

Open Access. This article is distributed under the terms of the Creative Commons Attribution License (CC-BY 4.0), which permits any use, distribution and reproduction in any medium, provided the original author(s) and source are credited.

\section{References}

[1] J.-L. Lehners, Ekpyrotic and cyclic cosmology, Phys. Rept. 465 (2008) 223 [arXiv:0806.1245] [INSPIRE]. 
[2] E. Silverstein and D. Tong, Scalar speed limits and cosmology: acceleration from D-cceleration, Phys. Rev. D 70 (2004) 103505 [hep-th/0310221] [INSPIRE].

[3] M. Alishahiha, E. Silverstein and D. Tong, DBI in the sky, Phys. Rev. D 70 (2004) 123505 [hep-th/0404084] [INSPIRE].

[4] D. Babich, P. Creminelli and M. Zaldarriaga, The shape of non-gaussianities, JCAP 08 (2004) 009 [astro-ph/0405356] [INSPIRE].

[5] J. Khoury, B.A. Ovrut, P.J. Steinhardt and N. Turok, The ekpyrotic universe: colliding branes and the origin of the hot big bang, Phys. Rev. D 64 (2001) 123522 [hep-th/0103239] [INSPIRE].

[6] R.Y. Donagi, J. Khoury, B.A. Ovrut, P.J. Steinhardt and N. Turok, Visible branes with negative tension in heterotic M-theory, JHEP 11 (2001) 041 [hep-th/0105199] [INSPIRE].

[7] S. Dubovsky, T. Gregoire, A. Nicolis and R. Rattazzi, Null energy condition and superluminal propagation, JHEP 03 (2006) 025 [hep-th/0512260] [INSPIRE].

[8] P. Creminelli, M.A. Luty, A. Nicolis and L. Senatore, Starting the universe: stable violation of the null energy condition and non-standard cosmologies, JHEP 12 (2006) 080 [hep-th/0606090] [INSPIRE].

[9] N. Arkani-Hamed, H.-C. Cheng, M.A. Luty and S. Mukohyama, Ghost condensation and a consistent infrared modification of gravity, JHEP 05 (2004) 074 [hep-th/0312099] [INSPIRE].

[10] N. Arkani-Hamed, H.-C. Cheng, M.A. Luty, S. Mukohyama and T. Wiseman, Dynamics of gravity in a Higgs phase, JHEP 01 (2007) 036 [hep-ph/0507120] [INSPIRE].

[11] M. Dine and N. Seiberg, Comments on higher derivative operators in some SUSY field theories, Phys. Lett. B 409 (1997) 239 [hep-th/9705057] [INSPIRE].

[12] M. Dine and J. Gray, Nonrenormalization theorems for operators with arbitrary numbers of derivatives in $N=4$ Yang-Mills theory, Phys. Lett. B 481 (2000) 427 [hep-th/9909020] [INSPIRE].

[13] A.A. Tseytlin and K. Zarembo, Magnetic interactions of D-branes and Wess-Zumino terms in super Yang-Mills effective actions, Phys. Lett. B 474 (2000) 95 [hep-th/9911246] [INSPIRE].

[14] D.V. Belyaev and I.B. Samsonov, Wess-Zumino term in the $N=4$ SYM effective action revisited, JHEP 04 (2011) 112 [arXiv:1103.5070] [INSPIRE].

[15] M. Henningson, Extended superspace, higher derivatives and $\mathrm{SL}(2, \mathbb{Z})$ duality, Nucl. Phys. B 458 (1996) 445 [hep-th/9507135] [INSPIRE].

[16] B. de Wit, M.T. Grisaru and M. Roček, Nonholomorphic corrections to the one loop $N=2$ super Yang-Mills action, Phys. Lett. B 374 (1996) 297 [hep-th/9601115] [INSPIRE].

[17] F. Gonzalez-Rey and M. Roček, Nonholomorphic $N=2$ terms in $N=4 S Y M$ : one loop calculation in $N=2$ superspace, Phys. Lett. B 434 (1998) 303 [hep-th/9804010] [INSPIRE].

[18] F. Gonzalez-Rey, B. Kulik, I.Y. Park and M. Roček, Selfdual effective action of $N=4$ super Yang-Mills, Nucl. Phys. B 544 (1999) 218 [hep-th/9810152] [InSPIRE].

[19] S.J. Gates, Jr., Why auxiliary fields matter: the strange case of the $4 D, N=1$ supersymmetric QCD effective action, Phys. Lett. B 365 (1996) 132 [hep-th/9508153] [INSPIRE]. 
[20] S.J. Gates, Jr., Why auxiliary fields matter: the strange case of the $4 D, N=1$ supersymmetric QCD effective action. 2, Nucl. Phys. B 485 (1997) 145 [hep-th/9606109] [INSPIRE].

[21] J. Khoury, J.-L. Lehners and B. Ovrut, Supersymmetric $P(X, \phi)$ and the ghost condensate, Phys. Rev. D 83 (2011) 125031 [arXiv:1012.3748] [inSPIRE].

[22] J. Khoury, J.-L. Lehners and B.A. Ovrut, Supersymmetric galileons, Phys. Rev. D 84 (2011) 043521 [arXiv:1103.0003] [InSPIRE].

[23] B. Ezhuthachan, S. Mukhi and C. Papageorgakis, The power of the Higgs mechanism: higher-derivative BLG theories, JHEP 04 (2009) 101 [arXiv:0903.0003] [INSPIRE].

[24] P.C. Argyres, A.M. Awad, G.A. Braun and F.P. Esposito, Higher derivative terms in $N=2$ supersymmetric effective actions, JHEP 07 (2003) 060 [hep-th/0306118] [INSPIRE].

[25] I.L. Buchbinder, E.A. Ivanov, I.B. Samsonov and B.M. Zupnik, Scale invariant low-energy effective action in $N=3 S Y M$ theory, Nucl. Phys. B 689 (2004) 91 [hep-th/0403053] [INSPIRE].

[26] I.L. Buchbinder and N.G. Pletnev, Construction of one-loop $N=4$ SYM effective action on the mixed branch in the harmonic superspace approach, JHEP 09 (2005) 073 [hep-th/0504216] [INSPIRE].

[27] I.L. Buchbinder and N.G. Pletnev, Hypermultiplet dependence of one-loop effective action in the $N=2$ superconformal theories, JHEP 04 (2007) 096 [hep-th/0611145] [INSPIRE].

[28] A. Gustavsson, Selfdual strings and loop space Nahm equations, JHEP 04 (2008) 083 [arXiv:0802.3456] [INSPIRE].

[29] A. Awad and M. Faizal, Higher-derivative terms for chiral superfields in four dimensions, in preparation.

[30] J. Bagger and N. Lambert, Comments on multiple M2-branes, JHEP 02 (2008) 105 [arXiv:0712.3738] [INSPIRE].

[31] J. Bagger and N. Lambert, Gauge symmetry and supersymmetry of multiple M2-branes, Phys. Rev. D 77 (2008) 065008 [arXiv:0711.0955] [INSPIRE].

[32] E. Antonyan and A.A. Tseytlin, On $3 D N=8$ Lorentzian BLG theory as a scaling limit of $3 D$ superconformal $N=6$ ABJM theory, Phys. Rev. D 79 (2009) 046002 [arXiv:0811.1540] [INSPIRE].

[33] O. Aharony, O. Bergman, D.L. Jafferis and J. Maldacena, $N=6$ superconformal Chern-Simons-matter theories, M2-branes and their gravity duals, JHEP 10 (2008) 091 [arXiv:0806.1218] [INSPIRE].

[34] M.A. Bandres, A.E. Lipstein and J.H. Schwarz, Studies of the ABJM theory in a formulation with manifest SU(4) R-symmetry, JHEP 09 (2008) 027 [arXiv:0807.0880] [INSPIRE].

[35] M. Schnabl and Y. Tachikawa, Classification of $N=6$ superconformal theories of ABJM type, JHEP 09 (2010) 103 [arXiv:0807.1102] [INSPIRE].

[36] O.-K. Kwon, P. Oh and J. Sohn, Notes on supersymmetry enhancement of ABJM theory, JHEP 08 (2009) 093 [arXiv:0906.4333] [InSPIRE].

[37] S. Sasaki, On non-linear action for gauged M2-brane, JHEP 02 (2010) 039 [arXiv: 0912.0903] [INSPIRE]. 
[38] F.S. Gama, M. Gomes, J.R. Nascimento, A.Yu. Petrov and A.J. da Silva, Generic higher-derivative $\mathcal{N}=2, D=3$ gauge theory, Phys. Rev. D 89 (2014) 085018 [arXiv: 1401.6839] [INSPIRE].

[39] F.S. Gama, J.R. Nascimento and A. Yu. Petrov, Effective superpotential in the generic higher-derivative three-dimensional scalar superfield theory, Phys. Rev. D 88 (2013) 065029 [arXiv: 1308.5834] [INSPIRE].

[40] S.J. Gates Jr., M.T. Grisaru, M. Rocek and W. Siegel, Superspace or one thousand and one lessons in supersymmetry, Front. Phys. 58 (1983) 1 [hep-th/0108200] [INSPIRE].

[41] A. Mauri and A.C. Petkou, An $N=1$ superfield action for M2 branes, Phys. Lett. B 666 (2008) 527 [arXiv:0806.2270] [INSPIRE].

[42] A. Gustavsson, One-loop corrections to Bagger-Lambert theory, Nucl. Phys. B 807 (2009) 315 [arXiv:0805.4443] [INSPIRE].

[43] D.B. Kaplan, Five lectures on effective field theory, nucl-th/0510023 [INSPIRE].

[44] C.P. Burgess, Introduction to effective field theory, Ann. Rev. Nucl. Part. Sci. 57 (2007) 329 [hep-th/0701053] [INSPIRE].

[45] R. MacKenzie, Path integral methods and applications, quant-ph/0004090 [INSPIRE].

[46] S. Coleman, Aspects of symmetry, Cambridge University Press, Cambridge U.K. (1985).

[47] M. Koehn, J.-L. Lehners and B.A. Ovrut, Higher-derivative chiral superfield actions coupled to $N=1$ supergravity, Phys. Rev. D 86 (2012) 085019 [arXiv:1207.3798] [INSPIRE]. 